\title{
Pregenerative medicine: developmental paradigms in the biology of cardiovascular regeneration
}

\author{
B. Alexander Yi, ${ }^{1}$ Oliver Wernet, ${ }^{1}$ and Kenneth R. Chien ${ }^{1,2}$ \\ ${ }^{1}$ Cardiovascular Research Center, Massachusetts General Hospital, Boston, Massachusetts, USA. 2Department of Stem Cell and Regenerative Biology, \\ Harvard University, Harvard Stem Cell Institute, Cambridge, Massachusetts, USA.
}

\begin{abstract}
The ability to create new functional cardiomyocytes is the holy grail of cardiac regenerative medicine. From studies using model organisms, new insights into the fundamental pathways that drive heart muscle regeneration have begun to arise as well as a growing knowledge of the distinct families of multipotent cardiovascular progenitors that generate diverse lineages during heart development. In this Review, we highlight this intersection of the "pregenerative" biology of heart progenitor cells and heart regeneration and discuss the longer term challenges and opportunities in moving toward a therapeutic goal of regenerative cardiovascular medicine.
\end{abstract}

The concept of self healing is ancient, capturing the imagination of generations of physicians and scientists over centuries. In modern times, this represents the reversal of the effects of a host of chronic human diseases - a major goal of regenerative medicine. One group of people for whom this quest is highly compelling is patients who have suffered myocardial injury. They face an incontrovertible reality: the heart, unlike the liver or skeletal muscle, is greatly limited in its capacity to regenerate itself. For instance, in a myocardial infarction roughly a billion or more cardiomyocytes are lost (1). The cardiomyocytes that survive are forced to work harder to maintain an adequate cardiac output. Over the long term, unable to keep up with increasing demands, the heart spirals downward in a cycle of adverse cardiac remodeling and neurohormonal activation that leads to congestive heart failure (2).

For the field of cardiovascular regenerative medicine, the scientific and clinical challenge is to develop novel therapeutic strategies that enhance the regeneration of normally functioning cardiac muscle in the failing heart. In this regard, exponential advances in stem cell and regenerative biology are beginning to foster a transition toward therapeutic goals for several important, unmet clinical needs. In the future, the convergence of stem cell biology with tissue engineering may usher in a new era of bioengineered muscle grafts, heart valves, blood vessels, and even entire hearts themselves (3-5). At present, the aim of most clinical trials has been to replenish the supply of functioning cardiomyocytes after a myocardial infarction and in those with chronic heart failure.

Conceptually, there are several ways of accomplishing this goal (Figure 1). The most obvious approach has been to simply transplant cells into the injured heart, with the hope that they might contribute to working cardiac muscle and thereby mitigate or reverse the progression of heart failure. In 2001, Orlic et al. reported that in a mouse model of myocardial infarction, bone marrow-derived c-Kit ${ }^{+}$cells transplanted into infarcted mouse hearts formed new cardiomyocytes and regenerated functional myocardium (6). This report spawned numerous clinical trials testing either the effect of transplanted bone marrow-derived cells or the administration of G-CSF in the acute myocardial infarction set-

Conflict of interest: The authors have declared that no conflict of interest exists. Citation for this article: J. Clin. Invest. 120:20-28 (2010). doi:10.1172/JCI40820. ting (7-14). The original report (6), however, was later called into serious question by other groups using more sensitive methods of detecting cellular transdifferentiation $(15,16)$. Indeed, there is a growing body of evidence suggesting the possibility that the early observations by Orlic and colleagues may have been the result of cell-cell fusion between transplanted cells and differentiated cardiac cells as well as the inherent limitations of relying on immunofluorescence markers alone as a readout compared with lineage-tracing tools (1, 17-20).

Nonetheless, early returns from human clinical trials suggest that delivering autologous bone marrow-derived mononuclear cells to infarcted regions of the heart may have marginal positive benefits (Table 1) $(21,22)$. The fortunate news is that the delivery of bone marrow-derived cells appears to have not led to serious adverse events that could have harmed further translational efforts; however, any improvement in left ventricular ejection fraction (approximately 3\% in a patient subset, where the control ejection fraction is around $50 \%$ in cumulative analysis of multiple trials) is most likely due to a still poorly understood paracrine effect $(23,24)$. While the modest size of effect and uncertainties surrounding the mechanism of action suggest that transplanting autologous bone marrow cells is not yet ready for widespread adoption, these early studies have illustrated the need for a better scientific understanding of the role of bone marrow-derived cells in myocardial remodeling or repair as well as a better understanding of how best to prepare and handle stem cells and how to optimize engraftment once they have been delivered to the heart (25). Since it is already widely accepted that positive inotropic effects (i.e., effects that increase the strength of muscle contraction) do not always translate into improved survival in the postinfarcted heart, particularly in the setting of heart failure (26), the question arises as to whether this marginal effect on ejection fraction will ultimately translate into an improvement in long-term survival, which will likely be required for any cell-based therapeutic protocol to achieve widespread acceptance as standard medical care.

Since the success of cardiovascular regenerative medicine may depend on identifying the optimal cell type to deliver to the heart, the notion that the adult heart may harbor stem cells with replicative and regenerative capacity has led some to search for autologous cardiac stem cells or progenitors from adult heart tissue. Although 


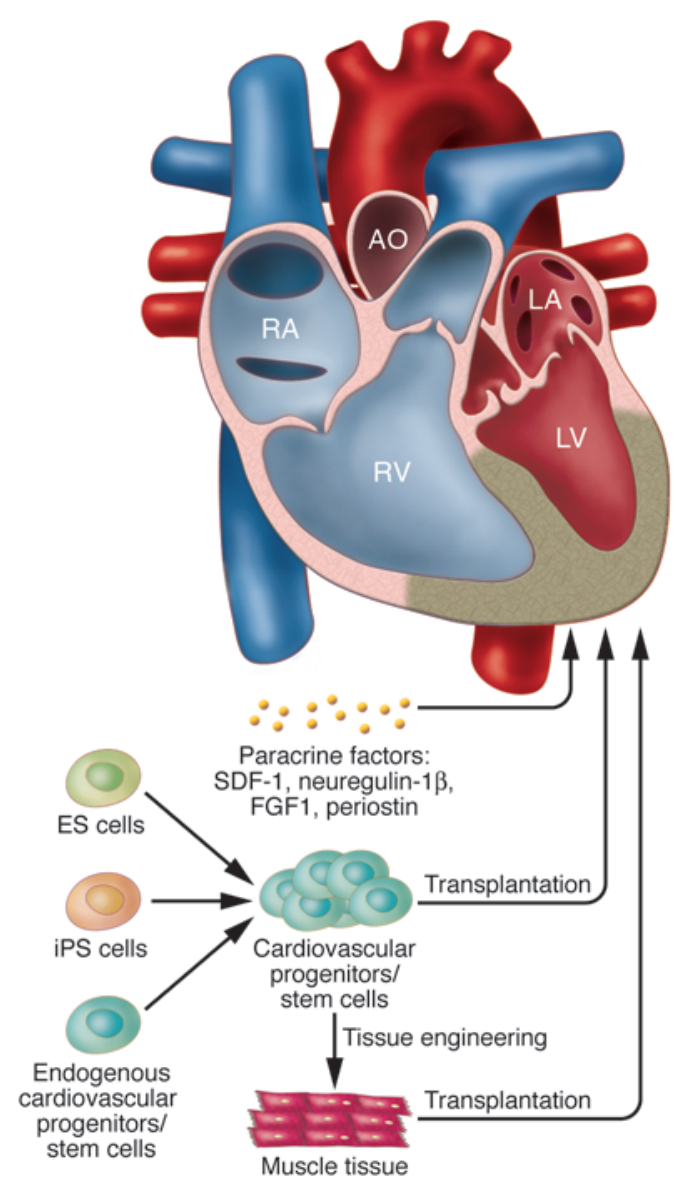

their exact lineage relationships are unclear, endogenous adult cardiac stem cells have been isolated in many species (including humans, mice, and rats) on the basis of stem cell markers such as stem cell antigen-1 (Sca-1) $(27)$ and c-Kit $(28,29)$ and of properties such as the ability to transport Hoechst dye (so called side population cells) $(30,31)$. These cell populations have been reported to be capable of proliferating and differentiating into cardiac myocytes. To date, undifferentiated human ES cells have only been tested in rodent models of heart disease $(32,33)$; however, the limitations of using human ES cells include inefficient cardiomyogenic differentiation, poor survival following transplantation, a tendency to form teratomas, and the risk of immunologic rejection (25).

Intriguingly, recent studies of heart regeneration now suggest another approach: enhancing the relatively limited endogenous regenerative capacity of the heart. The remarkable display of cardiac regeneration in some teleost and urodeles indicates that in nature, full cardiac regeneration is not unprecedented (34-36). Recent work has suggested that some degree of cardiomyocyte regeneration may occur even in mammals (37-39). Biological principles for cardiac regeneration are beginning to converge with recently uncovered pathways for cardiogenesis itself, linked via the emerging role of distinct families of multipotent cardiovascular progenitors and their downstream intermediates that generate diverse lineages during heart development. This Review highlights this recent intersection of the biology of heart regeneration and that of heart progenitor cells as well as the long-term challenges and opportunities in moving this toward a therapeutic goal.

\section{Figure 1}

Potential routes to cardiac regenerative medicine. Cardiovascular progenitors, generated from human ES cells or iPS cells or isolated from the patient's heart may be ideally suited for cardiac regenerative medicine since they are committed to the cardiac lineage and have the ability to self renew. They could be directly implanted into the heart or used to seed an extracellular matrix in order to engineer muscle tissue. Alternatively, the endogenous regenerative capacity of the heart may be stimulated by extracellular factors such as neuregulin-1 $\beta$. RA, right atrium; AO, aorta; LA, left atrium; SDF-1, stromal cell-derived factor 1.

\section{Principles of heart regeneration in model organisms}

For decades, scientists have marveled at the regenerative capacity of certain species. Our understanding of cardiac regeneration has been aided greatly by studies of the urodele newt and the teleost zebrafish, which are both able to regrow large portions of their hearts after amputation of the ventricle $(40,41)$. Grossly, the process seems similar in both animals. After amputation, a blood clot seals the injury site. Over the next few days, the clot matures into fibrin, and fibroblasts and a host of other inflammatory cells are attracted to the site. Then, starting from the edges of the wound, new cardiomyocytes are formed in a process that proceeds like a wave across the injury site until a new wall of myocardium is formed.

In cell culture, adult newt cardiomyocytes can divide to produce beating daughter cells, while in vivo studies suggest that upon injury, newt cardiomyocytes partially dedifferentiate into a progenitor-like state, expand, and then redifferentiate into cardiac cells $(34,35,42)$. These data fit with a paradigm that has been seen in other studies of organ or tissue regeneration in lower vertebrates (43-47). First, somatic cells at the injury site dedifferentiate into a multipotent state. Second, the multipotent cells proliferate in a localized region sometimes called the blastema. Finally, the multipotent cells redifferentiate back into the somatic cell type needed to regenerate the organ.

For zebrafish, however, the work of Lepilina and colleagues suggests that a pool of undifferentiated progenitors is the basis of cardiac regeneration (48). They used a strain of double-transgenic zebrafish that express both GFP and red fluorescent protein (RFP) under the control of the promoter of the cardiomyocyte-specific gene cardiac myosin light chain $(\mathrm{cmlc2})$. Their strategy relies on the fact that GFP has a shorter half-life than RFP because it folds and matures more quickly and is less stable $(49,50)$. Thus, newly formed cardiomyocytes from undifferentiated progenitors will express GFP but not RFP, while existing cardiomyocytes that dedifferentiate into a cardiac progenitor will generate cells that are predicted to be positive for RFP but not GFP. Interestingly, after cardiac amputation, a swath of $\mathrm{GFP}^{+} \mathrm{RFP}^{-}$cells was observed, consistent with the formation of new cardiomyocytes from undifferentiated progenitors. GFP-RFP ${ }^{+}$cells were never detected, suggesting that, unlike in the newt, cardiac regeneration in zebrafish may occur via a cardiac progenitor or precursor population. These results would interestingly suggest that the mechanisms of cardiac regeneration in the newt and the zebrafish are different. However, until conditional lineage-tracing experiments can be done to verify the origins of the cardiomyocytes formed during regeneration, it still remains an open question whether cardiac regeneration in zebrafish occurs predominantly through the recruitment of cardiac progenitors or through the dedifferentiation and replication of existing cardiomyocytes. 


\section{Table 1}

Randomized placebo-controlled trials of bone marrow-derived cells for ST elevation myocardial infarction

\begin{tabular}{|c|c|c|c|c|}
\hline Trial & $\begin{array}{c}\text { No. of } \\
\text { patients }\end{array}$ & Timing & Results & Reference \\
\hline ASTAMI & 100 & $\begin{array}{l}\text { Randomly assigned to receive intracoronary } \\
\text { bone marrow cells } 4 \text { to } 8 \text { days after Ml }\end{array}$ & $\begin{array}{l}\text { At } 6 \text { months: no effect on global LVEF } \\
\text { in group that received bone marrow cells }\end{array}$ & 10 \\
\hline \multirow[t]{2}{*}{ BOOST } & 60 & $\begin{array}{l}\text { Randomly assigned to receive intracoronary } \\
\text { bone marrow cells around } 5 \text { days after Ml }\end{array}$ & $\begin{array}{l}\text { At } 6 \text { months: } 6 \% \text { improvement in LVEF } \\
\text { in group that received bone marrow cells }\end{array}$ & 12,107 \\
\hline & & & $\begin{array}{l}\text { At } 18 \text { months: difference between groups } \\
\text { not significant }\end{array}$ & \\
\hline S. Janssens et al. & 67 & $\begin{array}{l}\text { Randomly assigned to receive intracoronary } \\
\text { bone marrow cells or placebo within } \\
24 \text { hours of reperfusion }\end{array}$ & $\begin{array}{l}\text { At } 4 \text { months: no significant improvement in } \\
\text { LVEF in group that received bone marrow cells }\end{array}$ & 9 \\
\hline J. Meluzin et al. & 66 & $\begin{array}{l}\text { Allocated to escalating doses of intracoronary } \\
\text { bone marrow cells } 3-4 \text { days or control }\end{array}$ & $\begin{array}{l}\text { At } 3 \text { months: } 6 \% \text { improvement in LVEF } \\
\text { in group that received a high dose } \\
\text { of bone marrow cells }\end{array}$ & 11 \\
\hline \multirow[t]{2}{*}{ REPAIR-AMI } & 204 & $\begin{array}{l}\text { Intracoronary bone marrow cells } \\
\text { or placebo } 3 \text { to } 7 \text { days after successful } \\
\text { reperfusion therapy for acute } \mathrm{MI}\end{array}$ & $\begin{array}{l}\text { At } 4 \text { months: absolute improvement in global } \\
\text { LVEF of } 2.5 \%(P=0.01) \text { in group that } \\
\text { received bone marrow cells }\end{array}$ & 14 \\
\hline & & & $\begin{array}{l}\text { At } 1 \text { year: reduction in combined clinical end point } \\
\text { of death, recurrent } \mathrm{MI} \text {, or any revascularization procedure } \\
\text { in group that received bone marrow cells }(P=0.01)\end{array}$ & \\
\hline TCT-STAMI & 20 & $\begin{array}{l}\text { Randomly assigned to receive bone } \\
\text { marrow cells or control in infarct-related } \\
\text { artery after PCl }\end{array}$ & $\begin{array}{l}\text { At } 6 \text { months: } 4.8 \% \text { improvement in LVEF in group } \\
\text { that received bone marrow cells; no change seen } \\
\text { in group that received control }\end{array}$ & 108 \\
\hline
\end{tabular}

LVEF, LV ejection fraction; MI, myocardial infarction; $\mathrm{PCl}$, percutaneous coronary intervention.

If indeed a cardiovascular progenitor or precursor is found in zebrafish, it is conceivable that similar progenitors may be at the root of cardiac regeneration in other species (Table 2). Despite the morphological differences between the zebrafish heart and the mammalian heart, the core cardiac transcription factors that direct heart development are evolutionarily conserved (51). For example, members of many human cardiac regulatory gene families, such as the homeobox gene NKX2-5, the LIM-homeodomain transcription factor ISLET1 (ISL1), myocyte enhancer factor 2 (MEF2), the T-box-containing transcription factor $T B X$, the zinc-finger transcription factor GATA4, and the basic helix-loop-helix transcription factor $H A N D$, are represented in zebrafish and may go as far back as Drosophila, Caenorhabditis elegans, and even some members of the phylum Cnidaria. Further work to determine the relationship of these putative progenitors to cardiovascular progenitors that have been recently isolated from mammalian hearts should prove of interest from a developmental as well as potentially therapeutic perspective (52-56).

\section{Cardiac regeneration in mammals}

Although it is commonly believed that the adult mammalian heart is devoid of the ability to generate new cardiomyocytes, reports going back decades have noted that mammalian cardiomyocytes do in fact have the capacity to undergo cell division, although this capacity is very limited $(57,58)$. Recent efforts to look for evidence of cardiomyocyte turnover in the adult mammalian heart have led to widely varying results that may be due in part to differences in the techniques used and the physiologic conditions studied $(38,58,59)$.

Hsieh and colleagues used transgenic mice to look at cardiomyocyte turnover using a pulse-chase approach (39). Specifi- cally, they engineered a transgenic mouse with a double-reporter system whereby cardiomyocytes expressed $\beta$-gal until tamoxifen was added. The addition of tamoxifen induced cardiomyocytes to turn off $\beta$-gal expression and instead turn on GFP expression. Tests of the double-reporter system indicated that with tamoxifen approximately $80 \%$ of cardiomyocytes became $\mathrm{GFP}^{+}$while the rest remained $\beta-\mathrm{gal}^{+}$. If there is cardiomyocyte turnover, then one would expect that over time the percentage of $\mathrm{GFP}^{+}$cardiomyocytes would decline as new cardiomyocytes expressing $\beta$-gal replaced them. If, however, there was no cardiomyocyte turnover, then the percentage of $\mathrm{GFP}^{+}$cardiomyocytes or the ratio of $\mathrm{GFP}^{+}$: $\beta$-gal ${ }^{+}$cells should remain constant. When the mice were analyzed a year after tamoxifen exposure, the percentage of $\mathrm{GFP}^{+}$cardiomyocytes was found to have remained constant, suggesting that during normal aging in the mouse, cardiomyocyte turnover does not occur. However, when these mice were subjected to experimental myocardial infarction or pressure overload and analyzed a few months later, the percentage of $\mathrm{GFP}^{+}$cardiomyocytes decreased substantially while the percentage of $\beta$-gal ${ }^{+}$myocytes increased, suggesting that new cardiomyocytes were generated in response to myocardial injury. The authors went on to postulate that the new cardiomyocytes may have been formed from a cardiac progenitor or precursor cell, because they had observed an increase in the expression of stem cell markers such as c-Kit and Nanog and had detected replicating $\mathrm{Nkx} 2.5^{+}$cells at myocardial infarction border areas (39). As with the zebrafish studies, conditional lineage-tracing studies will be required to unequivocally identify the origins of these putative progenitors and to directly quantitate their importance to the recovery of overall cardiac function following cardiac injury. 


\section{Table 2}

Pathways to cardiac regeneration

\begin{tabular}{lcccc} 
Animal & Adult/embryo & Injury model & Regenerative pathway & Reference \\
Newt & Adult & Mechanical injury & Cardiomyocyte dedifferentiation & 34,35 \\
Zebrafish & Adult & Amputation & Progenitor recruitment versus cardiomyocyte dedifferentiation & 36,48 \\
Rodent & Embryo & Genetic ablation & Cardiomyocyte proliferation; progenitor recruitment & 68 \\
Rodent & Adult & Experimental myocardial infarction; & Cardiomyocyte proliferation; progenitor recruitment & $39,65,67$ \\
Human & Adult & pressure overload & Cacardial infarction & Cardiomyocyte proliferation \\
\hline
\end{tabular}

Early pioneering studies by Beltrami and colleagues suggested that the human adult heart may also have an endogenous regenerative capacity (37). By staining human heart tissue with an antibody specific for Ki-67, a protein that is closely associated with the cell cycle and cell proliferation, they concluded that there is extensive cardiomyocyte proliferation during normal aging and that cardiomyocyte proliferation is markedly upregulated after a myocardial infarction. Compared with the number of $\mathrm{Ki}-67^{+}$cells in a series of control hearts from patients who died of noncardiac causes, they found that the number of Ki- $67^{+}$cells in hearts from patients who had experienced a myocardial infarction was 84 times higher in regions that bordered the infarct zone and 24 times higher in regions of the heart distant from the scar. Based on these data, they calculated a myocyte mitotic index and suggested that the billions of cardiomyocytes lost in a heart attack are all replaced in about 18 days, whereas under normal conditions, the entire set of myocytes in the heart is replaced about every 4.5 years (37). In other words, the entire pool of human cardiomyocytes was projected to be replaced approximately 18 times by the age of 80 (59).

In order to measure the rate of cardiomyocyte turnover in humans, Frisen and colleagues took advantage of a disturbing chapter in humankind's history of nuclear weapons (60). Between the mid-1950s and 1963, as a consequence of aboveground nuclear testing, atmospheric levels of ${ }^{14} \mathrm{C}$ rose dramatically. The ${ }^{14} \mathrm{C}$ quickly spread around the world and subsequently became incorporated into the food chain, eventually becoming part of the DNA of each individual cell. With the signing of the Partial Test Ban Treaty, levels of atmospheric ${ }^{14} \mathrm{C}$ dropped exponentially, back to near historical levels, but residual ${ }^{14} \mathrm{C}$ from those bomb blasts still persists in cells that were "born" during that era. Through remarkable work, Frisen has shown that levels of ${ }^{14} \mathrm{C}$ in a cell conform closely to ${ }^{14} \mathrm{C}$ levels in the atmosphere at the time the cell was born. Armed with this knowledge, they have successfully been able to calculate the lifespan of individual cells in the human body $(38,60-63)$.

Since the heart, like other tissues, is made up of several different cell types, Frisen and colleagues also had to invent methods of sorting cardiomyocyte DNA from the genomic DNA of other resident cardiac cell types, such as fibroblasts, that are known to be proliferative (38). They accomplished this by isolating nuclei from cardiac cells and then using antibodies to specifically label cardiomyocyte nuclei so that they could be sorted using a flow cytometer. Another challenge that Frisen and colleagues had to overcome is that in cardiomyocytes, DNA synthesis can occur independently of cell division since cardiomyocytes can be polyploid or binucleated (64). Taking these factors into account, they discovered that cardiomyocytes do indeed renew over the human lifespan, albeit at a very slow rate (38). This rate declines with age, so that at age 25 , about $1 \%$ of cardiomyocytes turn over annually, but at age 75 , that rate declines to $0.45 \%$ of the total cardiomyocytes. Over an average life span, this translates to about half of an individual's cardiomyocytes being exchanged.

One explanation for the discrepancy between the rate of turnover reported by Beltrami (37) and that determined using the ${ }^{14} \mathrm{C}$ method of dating cardiomyocytes (38) is the likelihood that the latter is inherently more quantitative than tissue-based assays that are dependent on the ability to discern cardiomyocytes from other cycling cardiac cells by immunofluorescence microscopy assays alone $(1,58)$. In addition, it has been pointed out that Ki-67 staining in the heart does not unequivocally establish that cell division has occurred, since, as mentioned above, a substantial proportion of cardiomyocytes are binucleated or polyploid and in the context of myocardial injury, polyploidy in cardiomyocytes may precede apoptosis (58). Further experimental resolution of some of these issues may be forthcoming in the near future via direct analysis of responses to injury in the human heart. The individuals studied by Frisen (38) had no documented history of cardiac disease, so it will be interesting to see if an increase in cardiomyocyte turnover in individuals with a history of heart disease is detected, as noted in the study of the murine heart by Hsieh (39).

If this endogenous regenerative potential can be harnessed by the administration of exogenous growth factors or cytokines, it may suggest an alternative to delivering cells directly to the heart to achieve cardiomyocyte regeneration or repair after myocardial infraction or in the failing heart. In a recent study, Kühn and colleagues demonstrated that administration of the EGF-like domain of neuregulin-1 $\beta$ (an agonist of the EGF family of receptor tyrosine kinases) can induce differentiated cardiomyocytes to reenter the cell cycle and undergo cell division in vivo (65). Furthermore, by expressing histone 2B-GFP under the control of the troponin $\mathrm{T}$ promoter, they were able to directly visualize differentiated cardiomyocytes as they underwent karyokinesis and/or cytokinesis by video microscopy. This visual evidence is important because one reason many have doubted the ability of cardiomyocytes to divide is the belief that their highly ordered myofibrils may physically prevent cytokinesis (64). By inducing cell division with neuregulin-1 $\beta$, Kühn and colleagues determined that cardiomyocytes undergoing cell division partially reorganize their sarcomeres to allow cytokinesis to occur. In the past, FGF1 and a component of the extracellular matrix known as periostin have also been demonstrated to induce DNA synthesis and cell division in differentiated cardiomyocytes in vitro and in vivo $(66,67)$. Interestingly, mononuclear cardiomyocytes were 


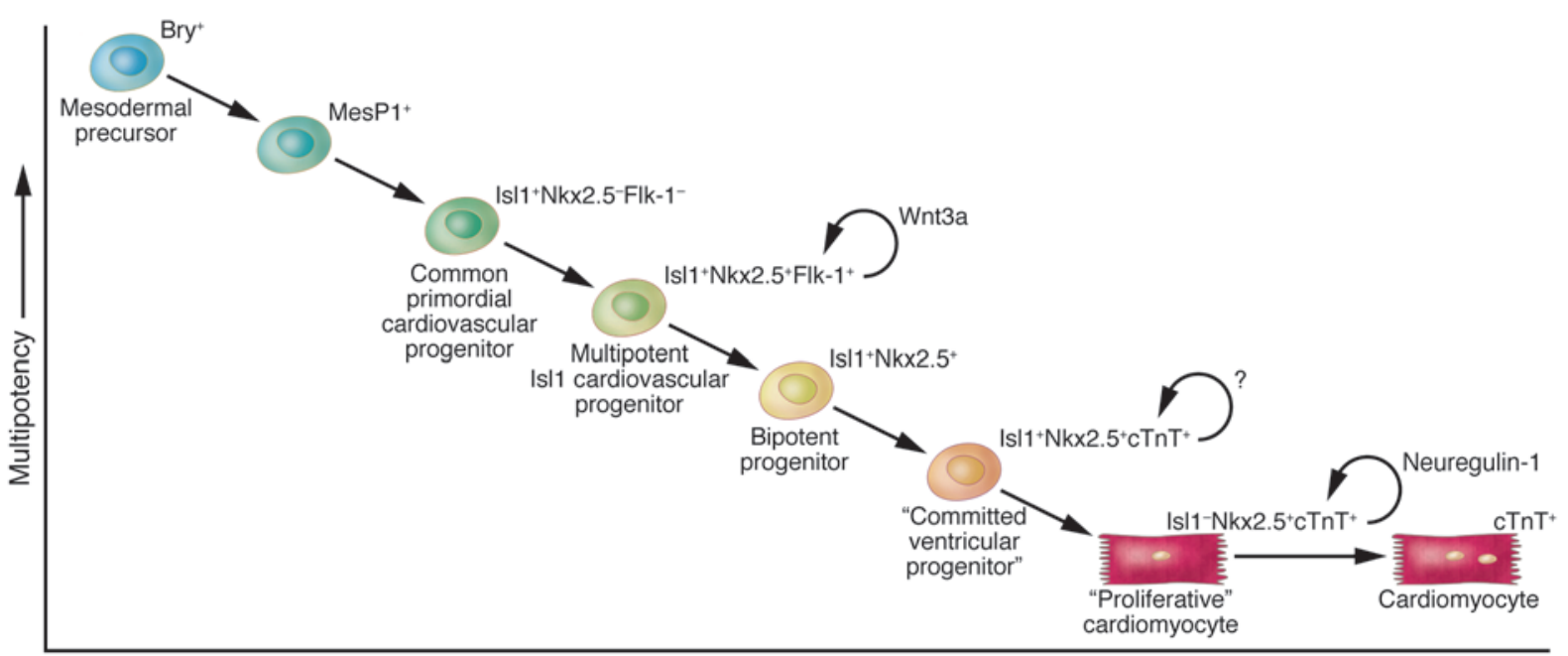

Differentiation

Figure 2

Proposed pathway for the differentiation of cardiomyocytes along the Is 1 lineage pathway. Differentiation proceeds from a mesodermal precursor cell to a common primordial cardiovascular progenitor that gives off a multipotent Isl1+ cardiovascular progenitor. Further differentiation proceeds via a committed ventricular progenitor to a terminally differentiated cardiomyocyte. cTnT, cardiac troponin T; MesP1, mesoderm posterior 1.

observed to undergo cell division but binucleated cardiomyocytes were not (65). After karyokinesis, about half the mononuclear cardiomyocytes were able to complete cytokinesis while the rest became a binucleated cardiomyocyte, suggesting an explanation for the gradually declining proliferative capacity of cardiomyocytes during normal aging $(38,65)$. Thus, while this work $(65-67)$ suggests that enhancing the endogenous regenerative capacity of the heart via extracellular factors is a potential approach to therapeutic cardiac regeneration, a limitation may be that the pool of cardiomyocytes that is capable of dividing gets smaller with age; one method of getting around this limitation may be to introduce cardiovascular progenitors that might increase the pool of cycling cardiomyocytes. Nonetheless, further study of the molecular pathways by which neuregulin- $1 \beta$ and periostin promote cardiomyocyte division may uncover compounds that may be useful therapeutically or serve as an adjunct to cell-based therapies. Also, further distinction between proliferating cardiomyocytes in the heart and other resident adult heart progenitors that have been described will be of interest.

\section{Studies of regeneration in the embryonic mammalian heart}

Studies of the mouse fetal heart suggest that an active cardiac proliferative response can compensate for the loss of a substantial number of cardiomyocytes (68). Not surprisingly, defects in the mitochondrial respiratory chain lead to severe cardiomyopathies (diseases characterized by deterioration of myocardial function), since cardiomyocytes are exquisitely sensitive to changes in metabolic conditions (69). In mammals, holocytochrome $c$ synthase, which is encoded by the gene Hccs on the X chromosome, converts cytochrome $c$ and cytochrome $c 1$ to their active forms by attaching a heme moiety (70). Drenckhahn and colleagues engineered a cardiac-specific Hccs knockout (68). As expected, hemizygous Hccs-knockout males and homozygous knockout females died in utero due to severe cardiomyopathic changes. Heterozygous females were also expected to display cardiomy- opathy, since roughly half their cardiomyocytes are lost due to random X chromosome inactivation. Surprisingly, however, the heterozygous females survived to birth and exhibited no major cardiac pathology. Examination of heterozygous females at different time points in gestation revealed that early in cardiogenesis heterozygous females indeed exhibited the expected 1:1 ratio of Hccs-deficient:Hccs-sufficient tissues. However, as gestation proceeded, the contribution of the diseased tissue to the heart gradually decreased, so that by birth, the proportion of diseased tissue represented only $10 \%$ of the cardiac tissue volume. Further analysis indicated that the healthy cells expanded and compensated for the loss of the Hccs-deficient cells. Is a cardiac progenitor involved? Most of the proliferating cells appeared to be cardiomyocytes, suggesting that fetal cardiomyocytes are able to readily reenter the cell cycle and divide; however, the authors could not discount the possibility that cardiac progenitors played a role, since not all the dividing cells could be positively identified as cardiomyocytes and some progenitors are known to persist into the late embryonic stages and even the postnatal heart (53).

Taken together, a growing body of evidence generated from the study of model organisms, mammalian model systems, and the human heart itself, is beginning to point to a need to understand the fundamental biology of heart progenitors, that is, to understand their origins, defining markers, renewal pathways, survival cues, triggers for differentiation into specific lineages, microenvironmental niche, and mechanisms of homing and migration to specific sites.

\section{The biology of mammalian cardiovascular progenitors}

The key features of a progenitor cell are clonality, self renewal, and often, multipotency. While the distinction between endogenous progenitors, transient cellular intermediates, and precursor cells might seem trivial, they represent distinct phases in the life of a heart cell (Figure 2) (71). Attempts to draw collective insight from an exponentially growing literature on mammalian heart stem cells from the adult and fetal heart, as well as from ES and induced pluripotent stem (iPS) cells, rest upon determining 
First heart field Second heart field Epicardial progenitors Cardiac neural crest

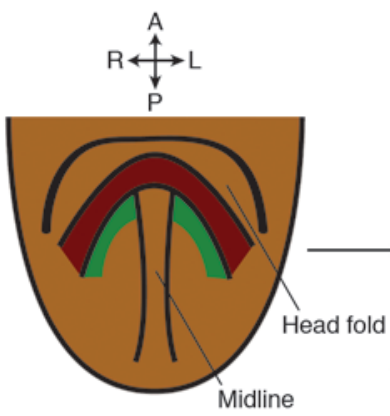

Head fold stage

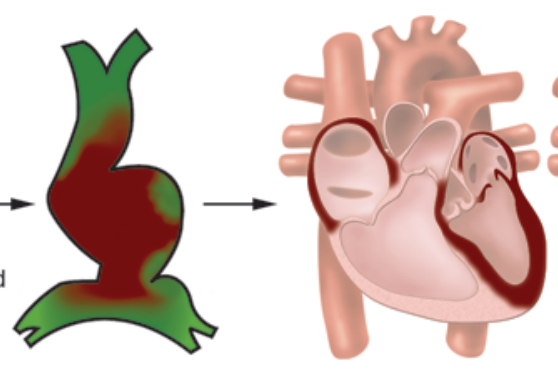

Heart tube stage

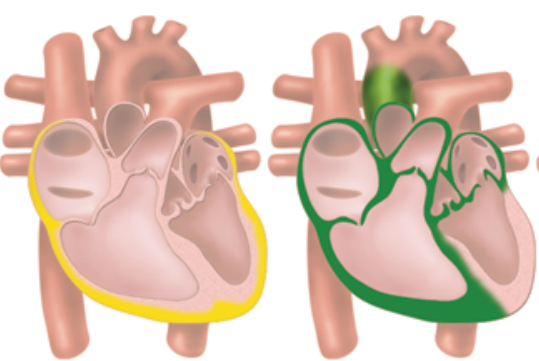

Four-chambered heart

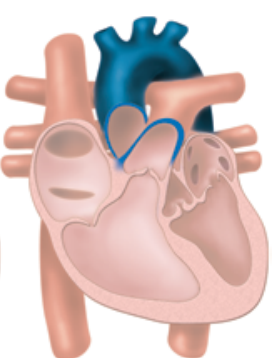

Figure 3

Mammalian heart development. The left panel depicts an early head fold stage. At this stage, the first heart field forms the cardiac crescent (red) while the second heart field (green) lies anterior and medial to the first heart field. The cells of the cardiac crescent join in the midline to form a linear heart tube. The middle panel depicts the heart tube as it begins to loop. Around this time, cells of the second heart field have migrated into the heart tube and will eventually contribute to cells of the atria, outflow tract, and right ventricle. Following rightward looping, cardiac neural crest cells migrate and contribute to the formation of the cardiac outflow tract. The right panel depicts the 4-chambered heart, denoting contributions from the first heart field, second heart field, epicardial progenitors, and cardiac neural crest.

criteria that establish that a given isolated cell of interest is an endogenous progenitor cell. Only in this way will it be possible to definitively compare studies of cardiovascular progenitors across different model systems (72).

Of course, one of the clearest ways to identify authentic cardiovascular progenitors is to establish their identity in the context of cardiogenesis itself and then to hunt for their existence in the adult heart under normal and pathological conditions. Identification of the origins of any cardiovascular progenitor in an embryologic context will be helpful in determining their physiologic role. Given the emerging role of cardiovascular progenitors in development, more recent data implying a role for them in regeneration in multiple vertebrate species, and their future potential in regenerative medicine, we provide a brief overview of known cardiovascular progenitors.

The heart is derived primarily from separate regions of mesoderm that are known as heart fields (73). The first, or primary, heart field is located bilaterally in the anterior splanchnic mesoderm and later undergoes a series of morphogenetic changes to form the cardiac crescent and then the linear heart tube (Figure 3). The first heart field ultimately gives rise to cells that contribute to both atria and the left ventricle. The second heart field is derived largely from pharyngeal mesoderm and is marked by expression of Isl1 (74). As the linear heart tube grows and undergoes looping, cardiovascular progenitors from the second heart field migrate into the heart tube and contribute cells that will form parts of the atria, the right ventricle, and the cardiac outflow tract (73). With the aid of gene-targeting methods, lineage tracing, and FACS analysis, multiple families of individual cardiovascular progenitor cells have been isolated by several laboratories $(52-56,75,76)$.

A common primordial cardiovascular progenitor that later gives rise to separate progenitors for the first and second heart fields has been postulated; however, the clear identification of this primordial cell has proved elusive $(71,77)$. From studies of in vitro differentiation of mouse ES cells, Kattman and colleagues used brachyury (Bry) and fetal liver kinase-1 (Flk-1), which encodes
VEGFR2, to identify a heterogeneous population of cells that exhibited cardiomyogenic potential (75). Upon ES cell differentiation, the first wave of Bry ${ }^{+} \mathrm{Flk}-1^{+}$cells corresponded to the hemangioblast, a population of mesodermal cells that differentiate into the hematopoietic and vascular lineages (78). A second wave of $\mathrm{Bry}^{+} \mathrm{Flk}-1^{+}$cells gave rise to colonies that were able to differentiate into cardiomyocytes, endothelial cells, and vascular smooth muscle cells (75). Some colonies became positive for the second heart field marker Isl1, while others were negative for Isl1 but expressed Tbx5, a marker associated with the first heart field (79). This suggests that this second Bry ${ }^{+} \mathrm{Flk}-1^{+}$population may be an early progenitor that precedes the separation of the first and second heart lineages. Correlating these cells with in vivo development, however, is challenging, since in vitro ES cell differentiation is highly dependent on culture conditions as well as exogenous factors that may not reflect the in vivo environment (56). More specific markers might be used to better isolate and delineate these early cardiovascular progenitors to sufficient purity to allow detailed analysis. For instance, during development, Flk-1 expression is continually turned on and off and has even been detected in undifferentiated ES cells, rendering Flk-1 a less than ideal marker for a cardiovascular progenitor $(52,80)$. In contrast, Isl1 becomes upregulated early during cardiogenesis and then becomes progressively downregulated upon cardiomyocyte differentiation $(54,74)$. These properties, and the specificity of Isl1 for the second heart field, have proven useful in helping to identify multipotent Isl $1^{+}$cardiovascular progenitors in both mouse and human $(52-54,74,81)$.

Wu and colleagues successfully isolated a third cardiovascular progenitor on the basis of expression of $\mathrm{Nkx} 2.5$, a homeobox transcription factor important for the development of ventricular cardiomyocytes (55). Both in vitro and in vivo, however, this progenitor underwent bipotential differentiation into cardiomyocytes and smooth muscle cells, suggesting that this bipotent progenitor lies downstream of the Bry ${ }^{+}$Flk- $1^{+}$progenitor $(56,75)$ and the Isl1 $1^{+}$ progenitor $(54,74)$. Of note, although $\mathrm{Nkx} 2.5$ is commonly associ- 
ated with the first heart field, Isl1 progenitors that go on to differentiate into cardiomyocytes also turn on $\mathrm{Nkx} 2.5$; therefore, additional markers will be necessary to clarify which heart field lineage the bipotent $\mathrm{Nkx} 2.5^{+}$progenitor belongs to (72). Since Nkx2.5 is essential for the development of ventricular cardiomyocytes (82), sorting isolated pools of cardiovascular progenitors for the subset that express $\mathrm{Nkx} 2.5$ might yield a fraction that is more committed to differentiate into cardiomyocytes (83).

Recently, epicardial progenitors were identified by expression of the transcription factor Wilms tumor 1 (Wt1) and Tbx18 and found to have cardiomyogenic potential $(76,84)$. Although epicardial progenitors mainly differentiate into smooth muscle cells and endothelial cells that contribute to the coronary vasculature, early in development, a subset of epicardial progenitors appear to differentiate into cardiomyocytes that contribute to all four chambers of the mouse heart $(76,84)$. The lineage origins of these epicardial progenitors remain to be determined, but data from Bin Zhou and colleagues suggest that $\mathrm{Wt} 1^{+}$epicardial progenitors may share a common origin with the cardiovascular progenitors described above, as they also express Isl1 and Nkx2.5 (76). The cardiomyogenic potential of these $\mathrm{Wt}^{+}{ }^{+}$epicardial progenitors is a novel finding and somewhat surprising. During zebrafish cardiac regeneration, the epicardial layer of cells around the heart becomes activated; however, in the zebrafish, the epicardial layer appears to contribute to neovascularization of the regenerate heart rather than to substantially contribute new cardiomyocytes (48). Interestingly, epicardial progenitors may only be able to differentiate into cardiomyocytes early in development. Cai and colleagues found that $\mathrm{Tbx} 18^{+}$proepicardial cells from embryos differentiated into cardiomyocytes in vitro, whereas $\mathrm{Tbx} 18^{+}$epicardial cells from postnatal mice did not (84).

Finally, the developmental origins of adult cardiac stem cells such as Sca- $1^{+}$cells $(27)$, c-Kit + cells $(28,29)$, and cardiac side population cells $(30,31)$ remain to be determined. They may belong to a pool of circulating stem cells that originate in the bone marrow or they may arise via mesoangioblasts from the surrounding vasculature $(27,85)$. A third possibility is that these cells are remnants of cardiogenesis (28). Defining the precise origin of these progenitors and their relationship to the embryonic progenitors noted above should prove of value to the field.

\section{The road behind: trials and tribulations of myoblast transfer}

Insights from the biology of skeletal muscle regeneration have the potential to carry over to cardiovascular regenerative medicine; trials of myoblast transfer offer a preview of the challenges that lie ahead $(86,87)$. The biology of skeletal muscle has several auspicious features that would suggest that regenerative therapies might be successful for treating disorders of skeletal muscle degeneration (87). For example, skeletal muscle is highly regenerative and known to harbor a population of resident muscle stem cells, which are known as satellite cells (88). In response to muscle injury, satellite cells, which normally sit dormant alongside the myofiber, become activated and differentiate into myoblasts that are capable of forming new muscle fibers or fusing with existing myofibers (89). A subpopulation of these satellite cells is also able to form new satellite cells that replenish the muscle stem cell pool $(90,91)$. Myoblasts can be readily expanded in vitro; therefore, it is possible to scale up production of a large number of cells for repeated cell transfers into skeletal muscle.
The lessons of myoblast transfer as a therapy for skeletal muscle regeneration suggest that several iterations will likely be necessary before the promise of both skeletal and cardiac muscle regenerative medicine can be realized. More than a decade ago, encouraging results in animal studies led researchers to attempt the first human trials of myoblast transfer for the treatment of Duchenne muscular dystrophy (DMD) (92). DMD arises from a loss-of-function mutation in dystrophin, an integral part of a complex that links the intracellular cytoskeleton with the extracellular matrix. The lossof-function mutation in dystrophin destabilizes this complex and renders skeletal muscles sensitive to cellular damage from repeated muscle contractions $(93,94)$. There are a multitude of approaches aimed at restoring dystrophin expression in muscle cells - a strategy demonstrated to be successful in improving muscle function in mouse models of the disease (95). Human trials with myoblasts have unfortunately yielded disappointing results (96-98). After injection of dystrophin-expressing myoblasts and repeat biopsy of the muscles, researchers detected low levels of dystrophin replacement in the muscles, but more importantly, the subjects demonstrated no improvement on tests of muscle contractility (98). The reasons for the failure of these trials is unclear, but investigators have directed their attention to a few critical issues (87). First, it was found that at least $75 \%$ of the transplanted cells died within the first few days (99). Second, the transplanted myoblasts remained localized around the injection site, rather than homing to sites of injury (100). Last, without appropriate immunosuppression, any surviving myoblasts were rapidly rejected within two weeks (101, 102). Nevertheless, more recent attempts at human myoblast transfer have achieved persistent dystrophin expression of more than $30 \%$ in injected muscle fibers, the best results thus far (103). It also seems that with this level of engraftment, improvements in muscle function might be accomplished (103).

Cardiac regenerative medicine is likely to face similar hurdles. In one instance, the biology of skeletal muscle regeneration has overlapped with cardiac regenerative medicine. Early attempts at cell-based therapies in the human heart involved injection of autologous skeletal myoblasts in and around regions of a myocardial scar in the hope that they might assist with myocardial performance and thereby prevent adverse cardiovascular remodeling (104). Following encouraging results from large animal studies, a randomized, placebo-controlled, double-blind study was done to assess the efficacy of delivering autologous skeletal myoblasts to patients with ischemic cardiomyopathy at the time of cardiac surgery (105). The primary end point was improvement in left ventricular ejection fraction at six months; however, results of this study showed that injections of skeletal myoblasts did not improve global or regional left ventricular function as compared with a placebo injection (106). While these studies have been informative, they also point out the need for alternative approaches for cardiovascular regenerative medicine.

\section{Conclusions}

The promise of regenerative medicine for severe forms of heart disease remains a truly noble goal that will ultimately require an interdisciplinary approach if it is to become a clinical reality. As such, major advances in cardiac regenerative medicine will likely occur in step with our growing understanding of the biology of heart regeneration and the molecular mechanisms that govern the self renewal and differentiation of cardiovascular progenitors as well as the development of new approaches to promote in vivo 
delivery, grafting, survival, maturation, and alignment of purified, well-characterized populations of human heart progenitors. Since there is evidence of a small degree of cardiomyocyte turnover in the adult mammalian heart, extracellular factors that can be administered to stimulate endogenous cardiomyocyte generation may be an interesting alternative approach or might be used in conjunction with cell-based approaches. Increasingly, a convergence of the pregenerative, degenerative, and regenerative biology of the heart should lay the groundwork for new therapeutic approaches for reversing key end points for cardiac diseases in the long term.

\section{Acknowledgments}

We would like to thank Lei Bu, Emil Hansson, Huansheng Xu, Silvia Martin-Puig, Yuan-hung Liu, Sean Wu, and members of the Chien laboratory for valuable feedback on the manuscript.

Address correspondence to: Kenneth R. Chien, Cardiovascular Research Center, Massachusetts General Hospital, Charles River Plaza/CPZN 3200, 185 Cambridge Street, Boston, Massachusetts 02114-2790, USA. Phone: (617) 643-3440; Fax: (617) 643-3451; E-mail: krchien@partners.org.
1. Laflamme MA, Murry CE. Regenerating the heart. Nat Biotechnol. 2005;23(7):845-856.

2. Sutton MG, Sharpe N. Left ventricular remodeling after myocardial infarction: pathophysiology and therapy. Circulation. 2000;101(25):2981-2988.

3. Grayson WL, Martens TP, Eng GM, Radisic M, Vunjak-Novakovic G. Biomimetic approach to tissue engineering. Semin Cell Dev Biol. 2009; 20(6):665-673.

4. Ott HC, et al. Perfusion-decellularized matrix: using nature's platform to engineer a bioartificial heart. Nat Med. 2008;14(2):213-221.

5. Yacoub MH, Takkenberg JJ. Will heart valve tissue engineering change the world? Nat Clin Pract Cardiovasc Med. 2005;2(2):60-61.

6 . Orlic D, et al. Bone marrow cells regenerate infarcted myocardium. Nature. 2001;410(6829):701-705.

7. Assmus B, et al. Transcoronary transplantation of progenitor cells after myocardial infarction. NEngl J Med. 2006;355(12):1222-1232.

8. Ince $\mathrm{H}, \mathrm{Nienaber} \mathrm{CA}$. Granulocyte-colony-stimulating factor in acute myocardial infarction: future perspectives after FIRSTLINE-AMI and REVIVAL-2. Nat Clin Pract Cardiovasc Med. 2007;4(suppl 1):S114-S118.

9. Janssens S, et al. Autologous bone marrow-derived stem-cell transfer in patients with ST-segment elevation myocardial infarction: double-blind, randomised controlled trial. Lancet. 2006;367(9505):113-121.

10. Lunde $\mathrm{K}$, et al. Intracoronary injection of mononuclear bone marrow cells in acute myocardial infarction. N Engl J Med. 2006;355(12):1199-1209.

11. Meluzin J, et al. Autologous transplantation of mononuclear bone marrow cells in patients with acute myocardial infarction: the effect of the dose of transplanted cells on myocardial function. Am Heart J. 2006;152:975.e979-975.e915.

12. Meyer GP, et al. Intracoronary bone marrow cell transfer after myocardial infarction: eighteen months' follow-up data from the randomized, controlled BOOST (BOne marrOw transfer to enhance ST-elevation infarct regeneration) trial. Circulation. 2006; 113(10):1287-1294.

13. Orlic D, et al. Mobilized bone marrow cells repair the infarcted heart, improving function and survival. Proc Natl Acad Sci U S A. 2001;98(18):10344-10349.

14. Schachinger $V$, et al. Intracoronary bone marrowderived progenitor cells in acute myocardial infarction. N Engl J Med. 2006;355(12):1210-1221.

15. Balsam LB, Wagers AJ, Christensen JL, Kofidis T, Weissman IL, Robbins RC. Haematopoietic stem cells adopt mature haematopoietic fates in ischaemic myocardium. Nature. 2004;428(6983):668-673.

16. Murry CE, et al. Haematopoietic stem cells do not transdifferentiate into cardiac myocytes in myocardial infarcts. Nature. 2004;428(6983):664-668.

17. Alvarez-Dolado M, et al. Fusion of bone-marrowderived cells with Purkinje neurons, cardiomyocytes and hepatocytes. Nature. 2003;425(6961):968-973.

18. Chien KR. Stem cells: lost in translation. Nature. 2004;428(6983):607-608.

19. Nygren JM, et al. Bone marrow-derived hematopoietic cells generate cardiomyocytes at a low frequency through cell fusion, but not transdifferentiation. Nat Med. 2004;10(5):494-501.
20. Terada N, et al. Bone marrow cells adopt the phenotype of other cells by spontaneous cell fusion. Nature. 2002;416(6880):542-545.

21. Hajjar R, Fuster V. Cardiac cell and gene therapies: two trajectories, one goal. Nat Clin Pract Cardiovasc Med. 2008;5:749.

22. Hansson EM, Lindsay ME, Chien KR. Regeneration next: toward heart stem cell therapeutics. Cell Stem Cell. 2009;5(4):364-377.

23. Korf-Klingebiel M, et al. Bone marrow cells are a rich source of growth factors and cytokines: implications for cell therapy trials after myocardial infarction. Eur Heart J. 2008;29(23):2851-2858.

24. Smith RR, Barile L, Messina E, Marban E. Stem cells in the heart: what's the buzz all about? Part 2: Arrhythmic risks and clinical studies. Heart Rhythm. 2008;5(6):880-887.

25. Segers VF, Lee RT. Stem-cell therapy for cardiac disease. Nature. 2008;451(7181):937-942.

26. Petersen JW, Felker GM. Inotropes in the management of acute heart failure. Crit Care Med. 2008; 36(suppl 1):S106-S111.

27. Oh $\mathrm{H}$, et al. Cardiac progenitor cells from adult myocardium: homing, differentiation, and fusion after infarction. Proc Natl Acad Sci U S A. 2003; 100(21):12313-12318.

28. Bearzi C, et al. Human cardiac stem cells. Proc Natl Acad Sci U S A. 2007;104(35):14068-14073.

29. Beltrami AP, et al. Adult cardiac stem cells are multipotent and support myocardial regeneration. Cell. 2003;114(6):763-776.

30. Goodell MA, et al. Dye efflux studies suggest that hematopoietic stem cells expressing low or undetectable levels of CD34 antigen exist in multiple species. Nat Med. 1997;3(12):1337-1345.

31. Hierlihy AM, Seale P, Lobe CG, Rudnicki MA, Megeney LA. The post-natal heart contains a myocardial stem cell population. FEBS Lett. 2002; 530(1-3):239-243.

32. Laflamme MA, et al. Cardiomyocytes derived from human embryonic stem cells in pro-survival factors enhance function of infarcted rat hearts. Nat Biotechnol. 2007;25(9):1015-1024.

33. van Laake LW, Passier R, Doevendans PA, Mummery CL. Human embryonic stem cell-derived cardiomyocytes and cardiac repair in rodents. Circ Res. 2008;102(9):1008-1010.

34. Bettencourt-Dias M, Mittnacht S, Brockes JP. Heterogeneous proliferative potential in regenerative adult newt cardiomyocytes. J Cell Sci. 2003; 116(Pt 19):4001-4009.

35. Laube F, Heister M, Scholz C, Borchardt T, Braun $\mathrm{T}$. Re-programming of newt cardiomyocytes is induced by tissue regeneration. J Cell Sci. 2006; 119(Pt 22):4719-4729.

36. Poss KD, Wilson LG, Keating MT. Heart regeneration in zebrafish. Science. 2002;298(5601):2188-2190.

37. Beltrami AP, et al. Evidence that human cardiac myocytes divide after myocardial infarction. NEngl JMed. 2001;344(23):1750-1757.

38. Bergmann O, et al. Evidence for cardiomyocyte renewal in humans. Science. 2009;324(5923):98-102.

39. Hsieh PC, et al. Evidence from a genetic fate-mapping study that stem cells refresh adult mam- malian cardiomyocytes after injury. Nat Med. 2007;13(8):970-974.

40. Borchardt T, Braun T. Cardiovascular regeneration in non-mammalian model systems: what are the differences between newts and man? Thromb Haemost. 2007;98(2):311-318.

41. Poss KD. Getting to the heart of regeneration in zebrafish. Semin Cell Dev Biol. 2007;18(1):36-45.

42. Curado S, Stainier DY. The HeArt of regeneration. Cell. 2006;127(3):462-464.

43. Kragl M, et al. Cells keep a memory of their tissue origin during axolotl limb regeneration. Nature. 2009;460(7251):60-65.

44. Kragl M, et al. Novel insights into the flexibility of cell and positional identity during urodele limb regeneration. Cold Spring Harb Symp Quant Biol. 2008;73:583-592.

45. Kumar A, Godwin JW, Gates PB, Garza-Garcia AA, Brockes JP. Molecular basis for the nerve dependence of limb regeneration in an adult vertebrate. Science. 2007;318(5851):772-777.

46. Morrison JI, Loof S, He P, Simon A. Salamander limb regeneration involves the activation of a multipotent skeletal muscle satellite cell population. J Cell Biol. 2006;172(3):433-440.

47. Poss KD, Keating MT, Nechiporuk A. Tales of regeneration in zebrafish. Dev Dyn. 2003;226(2):202-210.

48. Lepilina A, et al. A dynamic epicardial injury response supports progenitor cell activity during zebrafish heart regeneration. Cell. 2006;127(3):607-619.

49. Verkhusha VV, et al. High stability of Discosoma DsRed as compared to Aequorea EGFP. Biochemistry. 2003;42(26):7879-7884.

50. Verkhusha VV, Otsuna H, Awasaki T, Oda H, Tsukita S, Ito K. An enhanced mutant of red fluorescent protein DsRed for double labeling and developmental timer of neural fiber bundle formation. J Biol Chem. 2001;276(32):29621-29624.

51 . Olson EN. Gene regulatory networks in the evolution and development of the heart. Science. 2006; 313(5795):1922-1927.

52 . Bu L, et al. Human ISL1 heart progenitors generate diverse multipotent cardiovascular cell lineages. Nature. 2009;460(7251):113-117.

53. Laugwitz KL, et al. Postnatal isl1+ cardioblasts enter fully differentiated cardiomyocyte lineages. Nature. 2005;433(7026):647-653.

54. Moretti A, et al. Multipotent embryonic isl1+ progenitor cells lead to cardiac, smooth muscle, and endothelial cell diversification. Cell. 2006; 127(6):1151-1165.

55. Wu SM, et al. Developmental origin of a bipotential myocardial and smooth muscle cell precursor in the mammalian heart. Cell. 2006;127(6):1137-1150.

56 . Yang L, et al. Human cardiovascular progenitor cells develop from a KDR+ embryonic-stem-cell-derived population. Nature. 2008;453(7194):524-528.

57. Rumyantsev PP. Interrelations of the proliferation and differentiation processes during cardiact myogenesis and regeneration. Int Rev Cytol. 1977; 51:186-273.

58. Soonpaa MH, Field LJ. Survey of studies examining mammalian cardiomyocyte DNA synthesis. Circ Res. 1998;83(1):15-26. 
59. Anversa P, Kajstura J, Leri A, Bolli R. Life and death of cardiac stem cells: a paradigm shift in cardiac biology. Circulation. 2006;113(11):1451-1463.

60. Spalding KL, Bhardwaj RD, Buchholz BA, Druid $\mathrm{H}$, Frisen J. Retrospective birth dating of cells in humans. Cell. 2005;122(1):133-143.

61. Bhardwaj RD, et al. Neocortical neurogenesis in humans is restricted to development. Proc Natl Acad Sci U S A. 2006;103(33):12564-12568.

62. Spalding KL, et al. Dynamics of fat cell turnover in humans. Nature. 2008;453(7196):783-787.

63. Spalding KL, Buchholz BA, Bergman LE, Druid H, Frisen J. Forensics: age written in teeth by nuclear tests. Nature. 2005;437(7057):333-334

64. Ahuja P, Sdek P, MacLellan WR. Cardiac myocyte cell cycle control in development, disease, and regeneration. Physiol Rev. 2007;87(2):521-544.

65. Bersell K, Arab S, Haring B, Kühn B. Neuregulin1/ ErbB4 signaling induces cardiomyocyte proliferation and repair of heart injury. Cell. 2009;138(2):257-270.

66. Engel FB, et al. p38 MAP kinase inhibition enables proliferation of adult mammalian cardiomyocytes. Genes Dev. 2005;19(10):1175-1187.

67. Kuhn B, et al. Periostin induces proliferation of differentiated cardiomyocytes and promotes cardiac repair. Nat Med. 2007;13(8):962-969.

68. Drenckhahn JD, et al. Compensatory growth of healthy cardiac cells in the presence of diseased cells restores tissue homeostasis during heart development. Dev Cell. 2008;15(4):521-533.

69. Marin-Garcia J, Goldenthal MJ, Moe GW. Mitochondrial pathology in cardiac failure. Cardiovasc Res. 2001;49(1):17-26.

70. Bernard DG, Gabilly ST, Dujardin G, Merchant S, Hamel PP. Overlapping specificities of the mitochondrial cytochrome $\mathrm{c}$ and $\mathrm{c} 1$ heme lyases. J Biol Chem. 2003;278(50):49732-49742.

71. Martin-Puig S, Wang Z, Chien KR. Lives of a heart cell: tracing the origins of cardiac progenitors. Cell Stem Cell. 2008;2(4):320-331.

72. Chien KR, Domian IJ, Parker KK. Cardiogenesis and the complex biology of regenerative cardiovascular medicine. Science. 2008;322(5907):1494-1497.

73. Buckingham M, Meilhac S, Zaffran S. Building the mammalian heart from two sources of myocardial cells. Nat Rev Genet. 2005;6(11):826-835.

74. Cai CL, et al. Isl1 identifies a cardiac progenitor population that proliferates prior to differentiation and contributes a majority of cells to the heart Dev Cell. 2003;5(6):877-889.

75. Kattman SJ, HuberTL, Keller GM. Multipotent flk-1+ cardiovascular progenitor cells give rise to the cardiomyocyte, endothelial, and vascular smooth muscle lineages. Dev Cell. 2006;11(5):723-732.

76. Zhou B, et al. Epicardial progenitors contribute to the cardiomyocyte lineage in the developing heart.
Nature. 2008;454(7200):109-113.

77. Meilhac SM, Esner M, Kelly RG, Nicolas JF, Buckingham ME. The clonal origin of myocardial cells in different regions of the embryonic mouse heart. Dev Cell. 2004;6(5):685-698.

78. Huber TL, Kouskoff V, Fehling HJ, Palis J, Keller G. Haemangioblast commitment is initiated in the primitive streak of the mouse embryo. Nature. 2004;432(7017):625-630.

79. Takeuchi JK, et al. Tbx 5 specifies the left/right ventricles and ventricular septum position during cardiogenesis. Development. 2003;130(24):5953-5964.

80. Kouskoff V, Lacaud G, Schwantz S, Fehling HJ, Keller G. Sequential development of hematopoietic and cardiac mesoderm during embryonic stem cell differentiation. Proc Natl Acad Sci U S A. 2005; 102(37):13170-13175.

81. Qyang Y, et al. The renewal and differentiation of Isl1+ cardiovascular progenitors are controlled by a Wnt/beta-catenin pathway. Cell Stem Cell. 2007; 1(2):165-179.

82. Lyons I, et al. Myogenic and morphogenetic defects in the heart tubes of murine embryos lacking the homeo box gene Nkx2-5. Genes Dev. 1995; 9(13):1654-1666

83. Domian IJ, et al. Generation of functional ventricular heart muscle from mouse ventricular progenitor cells. Science. 2009;326(5951):426-429.

84. Cai CL, et al. A myocardial lineage derives from Tbx18 epicardial cells. Nature. 2008;454(7200):104-108

85. Dimmeler S, Zeiher AM, Schneider MD. Unchain my heart: the scientific foundations of cardiac repair. J Clin Invest. 2005;115(3):572-583.

86. Grounds MD, White JD, Rosenthal N, Bogoyevitch MA. The role of stem cells in skeletal and cardiac muscle repair. J Histochem Cytochem. 2002;50(5):589-610.

87. Peault B, et al. Stem and progenitor cells in skeletal muscle development, maintenance, and therapy. Mol Ther. 2007;15(5):867-877.

88. Mauro A. Satellite cell of skeletal muscle fibers. J Biophys Biochem Cytol. 1961;9:493-495.

89. Charge SB, Rudnicki MA. Cellular and molecular regulation of muscle regeneration. Physiol Rev. 2004;84(1):209-238.

90. Cerletti M, et al. Highly efficient, functional engraftment of skeletal muscle stem cells in dystrophic muscles. Cell. 2008;134(1):37-47.

91. Montarras D, et al. Direct isolation of satellite cells for skeletal muscle regeneration. Science. 2005; 309(5743):2064-2067.

92. Partridge T. Myoblast transplantation. Neuromuscul Disord. 2002;12 (suppl 1):S3-S6.

93. Ervasti JM, Ohlendieck K, Kahl SD, Gaver MG, Campbell KP. Deficiency of a glycoprotein component of the dystrophin complex in dystrophic muscle. Nature. 1990;345(6273):315-319.
94. Petrof BJ, Shrager JB, Stedman HH, Kelly AM, Sweeney HL. Dystrophin protects the sarcolemma from stresses developed during muscle contraction. Proc Natl Acad Sci U S A. 1993;90(8):3710-3714.

95. Cox GA, et al. Overexpression of dystrophin in transgenic mdx mice eliminates dystrophic symptoms without toxicity. Nature. 1993;364(6439):725-729.

96. Gussoni E, et al. Normal dystrophin transcripts detected in Duchenne muscular dystrophy patients after myoblast transplantation. Nature. 1992; 356(6368):435-438.

97. Karpati G, et al. Myoblast transfer in Duchenne muscular dystrophy. Ann Neurol. 1993;34(1):8-17.

98. Mendell JR, et al. Myoblast transfer in the treatment of Duchenne's muscular dystrophy. N Engl J Med. 1995;333(13):832-838.

99. Fan Y, Maley M, Beilharz M, Grounds M. Rapid death of injected myoblasts in myoblast transfer therapy. Muscle Nerve. 1996;19(7):853-860.

100.Ito H, Hallauer PL, Hastings KE, Tremblay JP. Prior culture with concanavalin A increases intramuscular migration of transplanted myoblast. Muscle Nerve. 1998;21(3):291-297.

101. Guerette B, Asselin I, Vilquin JT, Roy R, Tremblay JP. Lymphocyte infiltration following allo- and xenomyoblast transplantation in mice. Transplant Proc. 1994;26(6):3461-3462.

102.Guerette B, Asselin I, Vilquin JT, Roy R, Tremblay JP. Lymphocyte infiltration following allo- and xenomyoblast transplantation in $\mathrm{mdx}$ mice. Muscle Nerve. 1995;18(1):39-51.

103.Skuk D, et al. First test of a «high-density injection» protocol for myogenic cell transplantation throughout large volumes of muscles in a Duchenne muscular dystrophy patient: eighteen months follow-up. Neuromuscul Disord. 2007;17(1):38-46.

104. Minami E, Reinecke H, Murry CE. Skeletal muscle meets cardiac muscle. Friends or foes? J Am Coll Cardiol. 2003;41(7):1084-1086.

105. Menasche P, et al. Autologous skeletal myoblast transplantation for severe postinfarction left ventricular dysfunction. J Am Coll Cardiol. 2003; 41(7):1078-1083.

106. Menasche P, et al. The Myoblast Autologous Grafting in Ischemic Cardiomyopathy (MAGIC) trial: first randomized placebo-controlled study of myoblast transplantation. Circulation. 2008; 117(9):1189-1200.

107. Wollert KC, et al. Intracoronary autologous bonemarrow cell transfer after myocardial infarction: the BOOST randomised controlled clinical trial. Lancet. 2004;364(9429):141-148.

108. Ge J, et al. Efficacy of emergent transcatheter transplantation of stem cells for treatment of acute myocardial infarction (TCT-STAMI). Heart. 2006; 92(12):1764-1767. 